\title{
Antimicrobial Azaphilones from the Xylariaceous Inedible Mushrooms
}

\section{Dang Ngoc Quang, ${ }^{1}$ Toshibiro Hashimoto, ${ }^{1}$ Niko Radulovic, ${ }^{2}$ Marc Stadler, ${ }^{3}$ E Yoshinori Asakarwa ${ }^{1}$}

${ }^{1}$ Faculty of Pharmaceutical Sciences, Tokushima Bunri University, Yamashiro-cho, Tokushima 770-8514, Japan; ${ }^{2}$ Department of Chemistry, Faculty of Sciences and Mathematics, University of Niš Višgradska 33, 18000 Niš, Serbia and Montenegro; ${ }^{3}$ Bayer Health Care, PH-R\&D-R-EUET1, P.O. Box 101709, D-42096 Wuppertal, Germany

In the course of our investigation of biologically active substances from inedible mushrooms, we studied the chemical constituents of six species belonging to the Xylariaceae family (Sardariomycetideae) and isolated various types of azaphilone derivatives, which showed moderate to strong antimicrobial activity, such as entonaemins $\mathrm{A}-\mathrm{C}$ (1-3), together with (+)-mitorubrinol acetate (4), (+)-mitorubrin (5), (+)-mitorubrinol (6), and mitorubrinic acid (7) from Entonaema splendens (Hashimoto T. and Asakawa, 1998), daldinin C (8), daldinin E, F $(9,10)$ from Hypoxylon fuscum (Quang et al., 2004a), cohaerins A,B $(11,12)$ from $H$. cohaerens, rubiginosin A-C (13-15) from H.rubiginosum (Quang et al., 2004b), multiformins A-D (16-19) from $H$. multiforme, and sassafrins A-D (20-23) from Creospharea sassafras. Their absolute structures were elucidated by 2D NMR, MS, IR, $\mathrm{UV}, \mathrm{CD}$ spectra and chemical reaction (see figures on following page).

The in vitro antimicrobial activities of isolated azaphilones (1-23) at a dose of $50 \mu \mathrm{g}$ per paper disc were tested against a panel of laboratory control strains belonging to the American Type Culture Collection, Maryland, USA: Staphylococcus aureus, Klebsiella pneumoniae, Pseudomonas aeruginosa, Salmonella enteritidis, Escherichia coli 95, and fungal organisms Aspergillus niger and Candida albicans. The disc diffusion method, according to the NCCLS (National Committee for Clinical Laboratory Standards-Performance standards for antimicrobial disk susceptibility testing; $6^{\text {th }}$ International Supplement, Wayne Pa. 1997: M2-A6), was employed for the determination of the antimicrobial activity of the compounds. Table 1 (see next page) summarizes the antimicrobial properties of these azaphilones.

Accordingly, moderate to strong activity was observed against all tested strains. These effects, however, appear to be nonselective, because neither fungi nor bacteria remained unaffected by any of the azaphilone. They confirm previously reported bioactivities for other azaphilones and point toward the role of azaphilones as defense metabolites that may protect the stromata of Xylariaceae against feeding enemies or colonizing microbes.

\section{REFERENCES}

Hashimoto T. and Asakawa Y. 1998. Biologically active substances of Japanese inedible mushrooms. Het- erocycles, 47, 1067-1110.

Quang D. N., Hashimoto T., Tanaka M., Stadler M., and Asakawa Y. 2004a. Cyclic azaphilones daldinins $\mathrm{E}$ and $\mathrm{F}$ from the ascomycete fungus 
$\mathrm{HO}$<smiles>Cc1cc(C)c(C(=O)O[C@H]2CC3=C(CO2)C(=O)[C@](C)(O)[C@@]3(C)O)c(O)c1</smiles>

1. $\mathrm{R}=\mathrm{H}$

13. $R=A c$

$\mathrm{HO}$<smiles>Cc1cc(I)cc(O)c1C(=O)O[C@H]1CC2=C(COC([C@@H]3C[C@@H]3C)=C2)C(=O)[C@]1(C)O</smiles>

2<smiles>[R]C/C=C/C1=CC2=CC(=O)[C@@](C)(OC(=O)c3c(C)cc(O)cc3O)C(=O)C2=CO1</smiles>

4. $R=O A C$

5. $\mathrm{R}=\mathrm{H}$

6. $\mathrm{R}=\mathrm{OH}$

7. $\mathrm{R}=\mathrm{COOH}$<smiles>C/C=C(\C=C\C(=O)O[C@H]1C2=CC(=O)[C@@](C)(OC(C)=O)C(=O)C2=CO[C@]12O[C@@H](C)CC[C@H]2O)OC(C)=O</smiles>

10

Hypoxylon fuscum (Xylariaceae). Phytochemistry, 65, 469-473.

Quang D. N., Hashimoto T., Stadler, M., and Asakawa,<smiles>CC(=O)OC/C=C/C1=CC2=C(CO1)C(=O)[C@](C)(O)[C@@H](OC(=O)c1c(C)cc(Oc3cc(C)c(C(=O)O[C@H]4CC5=C(COC([C@H]6C[C@H]6C)=C5)C(=O)[C@]4(C)O)c(O)c3)cc1O)C2</smiles>

3<smiles>C/C=C(\C)C(=O)O[C@H]1C2=CC(=O)[C@@](C)(OC(C)=O)C(=O)C2=CO[C@]12O[C@H](C)CC[C@H]2O</smiles>

8<smiles>C/C=C/C=C(\C)C(=O)O[C@H]1C2=CC(=O)[C@@](C)(OC(C)=O)C(=O)C2=CO[C@]12O[C@H](C)CC[C@H]2O</smiles>

9

Y. 2004b. New azaphilones from the inedible mushroom Hypoxylon rubiginosum. J Nat Prod, 67, 1152-1155. 
<smiles>CCCCC/C=C(\C)C(=O)O[C@]1(C)C(=O)C=C2C=C(c3c(C)cccc3O)OC=C2C1=O</smiles>

11<smiles>CCCCC/C=C(\C)C(=O)O[C@H]1C(=O)C=C2C=C(C3=C(C)CC(O)CC3=O)OC=C2C1=O</smiles>

12<smiles>Cc1cc(O)cc(O)c1C(=O)O[C@]1(C)C(=O)C2=C(C=C(/C=C/CO)OC2)C[C@H]1O</smiles>

14<smiles>CCCCCCCCC/C=C/C=C(\C)C(=O)O[C@]1(C)C(=O)C=C2C=C(CC(C)O)OC=C2C1=O</smiles>

15<smiles>CCC(C)C(=O)[C@H]1C(=O)O[C@]2(C)C(=O)C=C3C=C(C4C(=O)C=CC[C@H]4C)OC=C3C=C12</smiles>

16<smiles>CCC(C)C(=O)C1=C2C3=COC(C4C(=O)C=CC[C@H]4C)=CC3=CC(=O)[C@@]2(C)OC1=O</smiles>

17<smiles>CCC(C)C(=O)[C@H]1C(=O)O[C@]2(C)C(=O)C=C3C=C(c4c(C)cccc4O)OC=C3C=C12</smiles>

18<smiles>CCC(C)C(=O)[C@H]1C(=O)O[C@]2(C)C(=O)C=C3C=C(C4C(=O)CC(O)C[C@H]4C)OC=C3C12</smiles><smiles>CCC(C)/C=C(C)/C=C/C(O)=C/C(=O)[C@H]1C(=O)O[C@]2(C)C(=O)C=C3C=C(CC(C)O)OC=C3[C@H]12</smiles>

20

19 
<smiles>CCC(C)/C=C/C=C/C(O)=C/C(=O)[C@H]1C(=O)O[C@]2(C)C(=O)C=C3C=C(CC(C)O)OC=C3[C@H]12</smiles>

21<smiles>CCC(C)/C=C(C)/C=C/C(=O)/C=C(\O)C1=C2C3=COC(CC(C)O)=CC3=CC(=O)[C@@]2(C)OC1=O</smiles>

22<smiles>CCC(C)/C=C/C=C/C(=O)c1cc2c(c(C(=O)O)c1O)C(C)(O)C(=O)C=C2CC(=O)CC(C)O</smiles>

23

Table 1. Antimicrobial Activity of Selected Azaphilones (1-23) (Diameter of the Zone of Growth Inhibition, Bactericidal, or Fungicidal Zone in $\mathrm{mm}$ ).

\begin{tabular}{|c|c|c|c|c|c|c|c|}
\hline Sample Microorganism & S. aureus & $\begin{array}{c}P . \\
\text { aeruginosa }\end{array}$ & $\begin{array}{c}\text { K. } \\
\text { pneumoniae }\end{array}$ & $\begin{array}{l}\text { S. } \\
\text { enteritidis }\end{array}$ & E. coli & A. niger & C. albicans \\
\hline Entonaemins A (1) & 18 & 7 & 13 & 7 & 8 & 14 & 14 \\
\hline DaldininC (8) & 7 & 7 & 8 & 8 & 7 & 14 & 15 \\
\hline Daldinin E (9) & 8 & 13 & 13 & 8 & 13 & 17 & 16 \\
\hline Daldinin F (10) & 14 & 7 & 7 & 7 & 7 & 16 & 15 \\
\hline Rubiginosin A (13) & 7 & 7 & 9 & 8 & 13 & 15 & 15 \\
\hline Rubiginosin B (14) & 13 & 16 & 15 & 15 & 19 & 16 & 16 \\
\hline Rubiginosin C (15) & 17 & 17 & 20 & 19 & 18 & 20 & 18 \\
\hline Multiformin A (16) & 17 & $0(+14)$ & 18 & 0 & 0 & $0(+19)$ & $0(+16)$ \\
\hline Multiformin B (17) & 18 & 19 & 20 & 19 & 19 & 18 & 19 \\
\hline Multiformin C (18) & 20 & 18 & 18 & 16 & 18 & 17 & 17 \\
\hline Multiformin D (19) & 18 & $0(+17)$ & 16 & $0(+16)$ & $0(+15)$ & 0 & $0(+20)$ \\
\hline Sassafrin A (20) & $0(+15)$ & 18 & 20 & 20 & 20 & 20 & 19 \\
\hline Sassafrin B (21) & 19 & 19 & 20 & 21 & 14 & 20 & 19 \\
\hline Sassafrin C (22) & 22 & 22 & 22 & 20 & 22 & 19 & 18 \\
\hline Sassafrin D (23) & 17 & 19 & 17 & 17 & 19 & 18 & 17 \\
\hline
\end{tabular}

